\title{
Accretion-induced spin-wandering effects on the neutron star in Scorpius X-1: Implications for continuous gravitational wave searches
}

\author{
Arunava Mukherjee, ${ }^{1,2,3, *}$ Chris Messenger, ${ }^{4, \dagger}$ and Keith Riles ${ }^{5,+}$ \\ ${ }^{1}$ Albert-Einstein-Institut, Max-Planck-Institut für Gravitationsphysik, D-30167 Hannover, Germany \\ ${ }^{2}$ Leibniz, Universität Hannover, D-30167 Hannover, Germany \\ ${ }^{3}$ International Centre for Theoretical Sciences, Tata Institute of Fundamental Research, \\ Bangalore 560012, India \\ ${ }^{4}$ SUPA, School of Physics and Astronomy, University of Glasgow, Glasgow G12 8QQ, United Kingdom \\ ${ }^{5}$ University of Michigan, Ann Arbor, Michigan 48109, USA
}

(Received 15 October 2017; published 28 February 2018)

\begin{abstract}
The LIGO's discovery of binary black hole mergers has opened up a new era of transient gravitational wave astronomy. The potential detection of gravitational radiation from another class of astronomical objects, rapidly spinning nonaxisymmetric neutron stars, would constitute a new area of gravitational wave astronomy. Scorpius X-1 (Sco X-1) is one of the most promising sources of continuous gravitational radiation to be detected with present-generation ground-based gravitational wave detectors, such as Advanced LIGO and Advanced Virgo. As the sensitivity of these detectors improve in the coming years, so will power of the search algorithms being used to find gravitational wave signals. Those searches will still require integration over nearly year long observational spans to detect the incredibly weak signals from rotating neutron stars. For low mass X-ray binaries such as Sco X-1 this difficult task is compounded by neutron star "spin wandering" caused by stochastic accretion fluctuations. In this paper, we analyze X-ray data from the RXTE satellite to infer the fluctuating torque on the neutron star in Sco X-1. We then perform a large-scale simulation to quantify the statistical properties of spin-wandering effects on the gravitational wave signal frequency and phase evolution. We find that there are a broad range of expected maximum levels of frequency wandering corresponding to maximum drifts of between $0.3-50 \mu \mathrm{Hz} / \mathrm{sec}$ over a year at $99 \%$ confidence. These results can be cast in terms of the maximum allowed length of a coherent signal model neglecting spin-wandering effects as ranging between 5-80 days. This study is designed to guide the development and evaluation of Sco X-1 search algorithms.
\end{abstract}

DOI: 10.1103/PhysRevD.97.043016

\section{INTRODUCTION AND MOTIVATION}

It has long been proposed that rapidly spinning neutron stars could emit detectable gravitational waves (GWs) [1-3] and that neutron stars in low-mass X-ray binary (LMXB) systems form an especially interesting class of objects. The neutron stars in these systems accrete matter from their companion stars ("donor") and thus can be spunup to spin frequencies $>500 \mathrm{~Hz}$ as the accreted matter also carries angular momentum to the neutron star [4], the

\footnotetext{
*arunava.mukherjee@aei.mpg.de

† Christopher.Messenger@glasgow.ac.uk

*kriles@umich.edu
}

Published by the American Physical Society under the terms of the Creative Commons Attribution 4.0 International license. Further distribution of this work must maintain attribution to the author(s) and the published article's title, journal citation, and DOI. amount of which is expected to be larger for larger mass accretion rates $(\dot{M})$. Observations show, however, that the fastest spinning accreting neutron star has a spin frequency much smaller than the expected maximum allowed value (breakup frequency) [5]. GWs have been proposed as a braking mechanism that prevents these accreting neutron stars from spinning up to higher frequencies $[5,6]$.

Recent detections of GWs from five confirmed binary black hole mergers and a binary neutron star merger have started a new era of transient GW astronomy [7-13]. In the contrasting continuous emission scenario, GWs generated from the neutron star within the Scorpius X-1 (Sco X-1) system are an exciting prospect for detection. Sco X-1 is the brightest persistent extra-solar $\mathrm{x}$-ray source in the sky. The high X-ray luminosity of Sco X-1 suggests an accretion rate close to the Eddington limit [14]. Moreover, the distance to the source is also quite small $\approx 2.8 \mathrm{kpc}$ [15]. Thus, it was identified early as a potential GW target [2]. In light of the 
recently upgraded second-generation GW detectors, Advanced LIGO and Advanced VIRGO, it should soon be possible to beat the "torque-balance" limit over a wide frequency range [16-18]. According to this scenario, the accretion-induced spin-up torque is primarily balanced by the total spin-down torque due to gravitational and electromagnetic (EM) radiation, although other braking mechanisms may also play a role [6].

The GW signals emitted from these rapidly spinning neutron stars will be extremely weak relative to the signals recently detected from binary black hole mergers [9], despite the relative proximity of galactic neutron star systems. The expected continuous GW strain-signal from a spinning neutron star in Sco X-1 will be of the order of $h \sim 10^{-25}$ or smaller [19]. Detection will likely require an observation duration of several months to years, with signal-to-noise ratio (SNR) accumulated via long term integration of the data.

One crucial practical problem in the search for GWs from LMXBs is spin wandering ( $\mathrm{SW})$, which can potentially degrade the effectiveness of continuous GW searches from accreting neutron stars. Although the spin-down torque due to $\mathrm{GW}$ and EM emission is generally unlikely to change by a significant amount during the observation period ( $\sim 1$ year), the mass accretion rate can change appreciably over this timescale, leading to an appreciable change in the instantaneous stellar spin-frequency and affect cumulative rotational phase. Such fluctuations make it challenging to integrate a GW signal coherently and achieve ideal detection efficiency. In this letter, we estimate for the first time detailed accretion fluctuation effects on the stellar spin frequency and on the cumulative rotational phase of the neutron star in the Sco X-1 system. The results can be applied to any other accreting neutron stars by choosing the values of appropriate physical parameters along with observational data from those systems.

In the following sections of this paper, we briefly describe our simulations to model astrophysically realistic spin-wandering effects on the continuous GW signals from the neutron star in Sco X-1. Numerical results are presented for expected fluctuation effects on the $\mathrm{GW}$ frequency $\left(f_{\mathrm{GW}}\right)$ and $\mathrm{GW}$ phase $\left(\phi_{\mathrm{GW}}\right)$ as a function of search integration time ranging over time scales of $\sim$ hour to greater than a year.

\section{FLUCTUATING TORQUE ON THE NEUTRON STAR AND THE SPIN WANDERING EFFECT}

Neutron stars in LMXB systems typically accrete matter from the companion star, often termed the "donor," through Roche-lobe overflow and are spun-up by the matter, a process known as "recycling" of neutron stars. According to the Bildsten et al. [4] model [see Eq. (7)], accretioninduced torque $(N)$ resulting changes in spin-frequency is given by,

$$
\begin{aligned}
\dot{f}_{\text {spin }}= & \left(\frac{N}{2 \pi I_{\text {spin }}}\right) \simeq 1.6 \times 10^{-13}\left(\frac{\dot{M}}{10^{-10} M_{\odot} \mathrm{yr}^{-1}}\right) \\
& \times\left(\frac{P_{\text {spin }}}{s}\right)^{1 / 3} \times\left(\frac{r_{m}}{r_{\text {co }}}\right)^{1 / 2} \times\left(\frac{I_{0}}{I_{\text {spin }}}\right) s^{-2},
\end{aligned}
$$

where $I_{\text {spin }}$ is the moment of inertia of the neutron star about the spin-axis, $I_{0}=10^{45} \mathrm{~g}-\mathrm{cm}^{2}, \dot{M}$ is the mass accretion rate, $P_{\text {spin }}=f_{\text {spin }}^{-1}$ is the spin-period, $r_{m}$ is the magnetic radius and $r_{\mathrm{co}}$ is the co-rotation radius of the accreting neutron star.

According to the torque balance scenario, accretion induced spin-up torque on the neutron star is balanced by the spin-down torques from EM and gravitational radiation $[3,5]$. The spin-down torque due to EM radiation depends mainly on the magnetic dipole field of the neutron star, and that due to gravitational radiation depends on the quadrupolar deformation of the star. Neither the external magnetic field nor the quadrupolar deformation, ignoring time varying excitations such as r-mode [20,21], is likely to change significantly over our longest considered timescale of $\sim 1$ yr. Hence we neglect changes in spin-down torques in the following analysis.

The mass accretion rate $(\dot{M})$ for a neutron star in a typical LMXB system (including Sco X-1) can change appreciably on much shorter timescales than $\sim 1$ yr. The instantaneous mass accretion rate $\dot{M}(t)$ can thus be split into two parts:

$$
\dot{M}(t)=\langle\dot{M}(t)\rangle+\Delta \dot{M}(t) .
$$

However, $\dot{M}(t)$ is related to the amount of instantaneous torque and thus to the spin frequency derivative $\dot{f}(t)$ of the neutron star according to Eq. (2), that derivative can be decomposed:

$$
\dot{f}(t)=\langle\dot{f}(t)\rangle+\Delta \dot{f}(t) .
$$

The torque balance scenario asserts that, over a long timescale, the spin-up and spin-down torques balance each other, i.e., $\langle\dot{f}(t)\rangle=0$; but $\Delta \dot{f}(t) \neq 0$ over a time scale of hours to a few years, because of the observed $\Delta \dot{M}(t) \neq 0$ (see Fig. 1).

As a consequence, the neutron star is out of spinequilibrium instantaneously and either spinning up or spinning down as the resultant torque fluctuates. We call the corresponding fluctuation in spin-frequency relative to the long term average, the "spin-wandering" effect. In this paper, we perform a first attempt to estimate quantitatively this spin-wandering effect for the neutron star in Sco X-1 over relevant GW search timescales and using an astrophysically-motivated model together with relevant observational data.

For the purpose of estimating the spin-wandering effect, we therefore require information regarding the time variation of the mass accretion rate $(\dot{M}(t))$, the spin period $\left(P_{\text {spin }}\right)$, and the magnetic and corotation radii $\left(r_{m}\right.$ and $\left.r_{\mathrm{co}}\right)$ of 

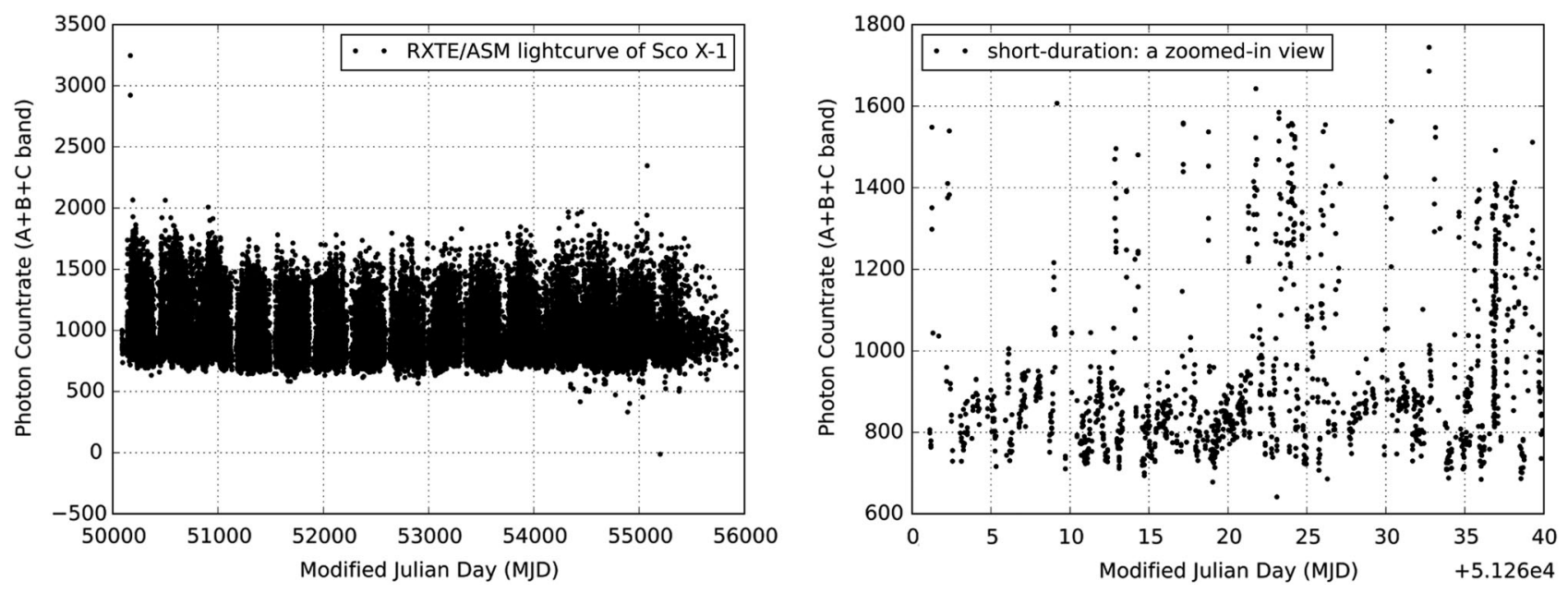

FIG. 1. Left panel: Sum-band (i.e., A + B + C bands) photon count-rates over nearly 15 years of RXTE/ASM monitoring of Sco X-1. The ASM sum-band covers the photon energy range of 2-10 keV. Right panel: Magnification of one sample 40-day period in the left panel.

the neutron star. We discuss estimates for these quantities in the subsequent sections.

\section{A. Estimating the mass accretion rate: Analyzing x-ray data}

Estimating the time-dependent mass accretion rate $\dot{M}(t)$ is an important part of this analysis. The x-ray flux from an accreting LMXB is taken as an estimator of instantaneous $\dot{M}$, provided we know the distance to the source [22]. The distance to Sco X-1 has been reported to be $2.8 \pm 0.3 \mathrm{kpc}$ [15], and data from the long term monitoring of Sco X-1 can be utilized to estimate the character of the fluctuations $\Delta \dot{M}(t)$. For this purpose we used archival x-ray data from the all-sky monitor (ASM) instrument of Rossi X-ray Timing Explorer (RXTE). ${ }^{1}$ For the purpose of estimating the time-averaged mass accretion rate $\langle\dot{M}(t)\rangle$, and the time dependent variability $\Delta \dot{M}(t)$ around this mean value, we performed the following steps.

We analyzed the timeseries of the sum-band (i.e., A + $\mathrm{B}+\mathrm{C}$ bands) photon count-rates of Sco $\mathrm{X}-1$ from the $R X T E / A S M$ instrument giving us a crude estimate of $\langle\dot{M}(t)\rangle$ and its fluctuation over a period of nearly 15 years. The resultant RXTE/ASM lightcurve is shown in Fig. 1. From the two panels of the figure, one can see that the mass-accretion rate fluctuates quite dramatically over a wide range of time scales. The scale of fluctuation in luminosity and correspondingly in the inferred $\dot{M}$ often become comparable to their respective mean values, suggesting that fluctuations in accretion induced torque, and consequently the spin-wandering effect, may be significant.

This sum-band photon count-rate of RXTE/ASM alone, however, cannot be used to infer the $\dot{M}(t)$, as (i) the does not have a good energy resolution and thus cannot provide

\footnotetext{
${ }^{1}$ for details see https://heasarc.gsfc.nasa.gov/docs/xte/xte_1st .html.
}

x-ray flux accurately, and (ii) it detects only photons in the $2-10 \mathrm{keV}$ range. For this purpose, we perform the following steps. Since, Sco X-1 is a highly variable source, we cannot compare the flux across different bands observed at different epochs. Therefore, we compute the 95\% confidence level upper-limit and lower limit of the RXTE/ASM count-rate to be 1716 photons/s and 618 photons/s, respectively. Comparing the reported upper-limit of mass accretion rate from Sco X-1 by McNamara et al. [23], we find the upper-limit (95\% confidence level) of the flux corresponds to $\simeq 0.42 \times 10^{-8} \mathrm{M}_{\odot} \mathrm{yr}^{-1}$ and lower-limit (95\% confidence level) of the flux corresponds to $\simeq 1.2 \times 10^{-8} \mathrm{M}_{\odot} \mathrm{yr}^{-1}$. This corresponds to a conversion factor of $7.03 \times 10^{-10} \mathrm{M}_{\odot} \mathrm{yr}^{-1}$ per 100 photon count-rate in RXTE/ASM.

In parallel, we make an attempt to estimate the X-ray flux from Sco X-1. In order to estimate it, we need to analyze spectral data from an instrument with good photon energy resolution. Since, the spectral resolution of the ASM instrument is limited, we use another instrument, the Proportional Counter Array (PCA) ${ }^{2}$ from the same satellite as a calibration reference. The PCA enables us to find a number of (nearly) simultaneous measurements of Sco X-1 and allows us to estimate the energy flux in $2-10 \mathrm{keV}$ from the measured $\mathrm{x}$-ray spectra.

We analyzed a few pointed RXTE/PCA observations that are (near) simultaneous to some of the ASM observations of Sco X-1. We performed the data extraction using the standard FTOOLs ${ }^{3}$ analysis software and background subtraction using runpcabackest ${ }^{4}$ tool in the

\footnotetext{
${ }^{2}$ for details see https://heasarc.gsfc.nasa.gov/docs/xte/PCA .html.

${ }^{3}$ for details see https://heasarc.gsfc.nasa.gov/ftools/ftools_ menu.html.

${ }^{4}$ for details see https://heasarc.gsfc.nasa.gov/docs/xte/recipes/ pcabackest.html.
} 


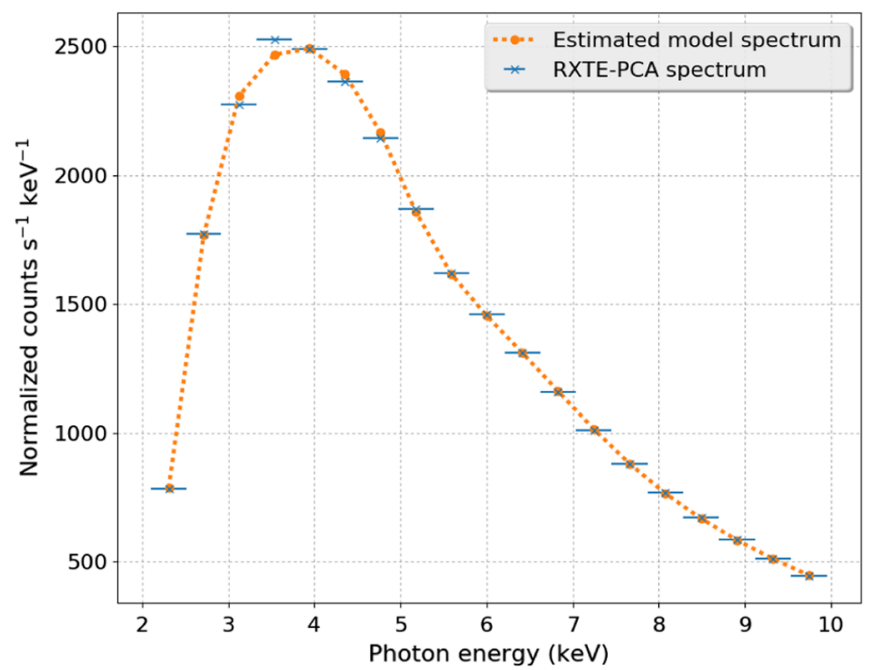

FIG. 2. The spectral energy distribution of Sco X-1 in the photon energy band of 2-10 keV observed with the RXTE/PCA instrument. The observed spectrum is well described by a combination of phabs* (diskbb+compTT+Gaussian). The discrete (light-blue colored) lines represent data from RXTE/PCA and the dotted continuous (oranged colored) lines are the estimated model spectrum. We have performed this model estimation and corresponding x-ray flux using the xspec spectral analysis package.

HEASOFT $^{5}$ data analysis software. In each case, the continuum spectral energy density (SED) in the 2-10 keV photon energy band can be described well with a combination of the phabs * (diskbb+compTT+Gaussian) model $^{6}$ using the $\mathrm{xspec}$ spectral analysis package ${ }^{7}$ to obtain satisfactory $\left(\chi^{2} / \text { dof } \approx 1\right)^{8}$ statistical significance (see Fig. 2). From the best-fit models, we have estimated the fluxes in the analyzed $2-10 \mathrm{keV}$ bands. The observed fluxes scale well (to within $\sim 10 \%$ ) with the sum-band photon count-rates from simultaneous RXTE/ASM observations. In Fig. 2 we show a representative plot with the best-fit theoretical model along with the corresponding $R X T E / P C A$ observations. From Fig. 2, we see that most of the X-ray flux of Sco X-1 comes in this $2-10 \mathrm{keV}$ photon energy range. Thus, it can be taken as a good tracer of $\dot{M}(t)$, shown in Fig. 3.

However, the estimated flux from this photon energy spectrum corresponds to the emission only in the $2-10 \mathrm{keV}$ band, whereas we need to measure the bolometric flux from Sco X-1 to estimate the actual $\dot{M}$. In order to achieve this, we estimate the theoretical flux over a sufficiently large range, $0.1-50.0 \mathrm{keV}$, over which most of the radiation from Sco X-1 is captured, and then use it to scale the time-series

\footnotetext{
${ }^{5}$ for details see https://heasarc.gsfc.nasa.gov/lheasoft/.

${ }^{6}$ for details of each individual model see https://heasarc.gsfc .nasa.gov/xanadu/xspec/manual/XspecModels.html.

${ }^{7}$ for details of the spectral analysis package see https://heasarc .gsfc.nasa.gov/xanadu/xspec/manual/manual.html.

${ }^{8}$ dof stands for number of degrees of freedom.
}

of $R X T E / A S M$ count-rates. The estimated flux in the $0.1-50.0 \mathrm{keV}$ energy band from these observations is found to be $\simeq 3.56 \times 10^{-7} \mathrm{erg} \mathrm{cm}^{-2} \mathrm{~s}^{-1}$, which corresponds to $5.9 \times 10^{-9} \mathrm{M}_{\odot} / \mathrm{yr}$ for a source distance of $2.8 \mathrm{kpc}$. The corresponding (near) simultaneous ASM photon count-rate corresponds to $956 \mathrm{~s}^{-1}$. This estimation of $\dot{M}(t)$ of Sco X-1 from $R X T E / \mathrm{PCA}$ is in good agreement with (only $14 \%$ below) the corresponding estimation from the ASM countrate calibrated to McNamara et al. [23]. This underestimate in $\dot{M}(t)$ could, possibly, due to presence of some other emission mechanisms predominant outside this $2-10 \mathrm{keV}$ band over which we modeled photon energy spectrum which can result in a small fraction of the flux falling outside this measured energy band. It is worth mentioning that, the uncertainty in distance to Sco $\mathrm{X}-1$ is $\sim 10 \%$ implying an uncertainty in absolute flux of $\sim 20 \%$. For our purpose, this uncertainty in $\dot{M}(t)$ is rather small as compared to other uncertainties in our modelling as discussed in Sec. IV.

\section{B. Estimating magnetic radius $\left(r_{m}\right)$ and corotation radius $\left(r_{\mathrm{co}}\right)$}

The magnetic radius $\left(r_{m}\right)$ and corotation radius $\left(r_{\mathrm{co}}\right)$ play crucial roles too, in determining the spin-wandering effect for an accreting neutron star in an LMXB system (see Eq. (1)). The ratio of these two characteristic radii, $\left(r_{m} / r_{\mathrm{co}}\right)$, determines the coupling between the magnetosphere of the neutron star and the accretion disk around it. The magnetic radius length-scale corresponds to $r_{m}=\xi r_{\mathrm{A}}$; where $\xi$ is expected to be in the range $\simeq 0.5-1$. It is not completely certain how much the accreted matter is guided by the field lines to fall on to the polar caps. In this paper we take a conservative approach, leading to the maximum possible spin-wandering effect. Here, $r_{\mathrm{A}}$ is the Alfvén radius, quantified in this case as:

$$
\begin{aligned}
r_{\mathrm{A}}= & \left(\frac{\mu^{4}}{2 G M \dot{M}^{2}}\right)^{1 / 7} \simeq 6.8 \times 10^{8}\left(\frac{\mu}{30 \mathrm{Gcm}^{3}}\right)^{4 / 7} \\
& \times\left(\frac{10^{-10} \mathrm{M}_{\odot} \mathrm{yr}^{-1}}{\dot{M}}\right)^{2 / 7} \times\left(\frac{1.4 \mathrm{M}_{\odot}}{M}\right)^{1 / 7} \mathrm{~cm},
\end{aligned}
$$

where $\mu$ is the star's magnetic dipole moment and $M$ is the mass of the neutron star.

This Alfvén radius $\left(r_{\mathrm{A}}\right)$ is reasonably well determined, provided we know the neutron star's magnetic field $\left(\mathrm{B}_{\mathrm{NS}}\right)$ and the mass-accretion rate $(\dot{M})$. For Sco X-1, from X-ray observations (see above) we have estimated that $\dot{M} \simeq 0.4-1.2 \times 10^{-8} \mathrm{M}_{\odot} \mathrm{yr}^{-1}$, but the strength of the magnetic field is less well known. The absence of any strong and persistent pulsation in Sco X-1, however, and the fact that Sco X-1 is an LMXB system suggests that the external magnetic field $B_{\mathrm{NS}}$ is less than $\sim 10^{12} \mathrm{G}$. This estimate is very conservative; a more realistic estimate is $B_{\mathrm{NS}} \leq 10^{10} \mathrm{G}$ $[24,25]$. In the other extreme, the minimum observed field values for neutron stars are $B_{\mathrm{NS}} \simeq 10^{7}-10^{8} \mathrm{G}[24,25]$. 
Considering the plausible uncertainties in $M$ and $\mathrm{M}_{\odot}$ for the neutron star in Sco $\mathrm{X}-1$, this range corresponds to $r_{m} \simeq 10-2000 \mathrm{~km}$.

The other characteristic length-scale, the corotation radius $\left(r_{\mathrm{co}}\right)$ is quantified from Kepler's 3rd Law as:

$$
\begin{aligned}
r_{\mathrm{co}}= & \left(\frac{G M P_{\text {spin }}^{2}}{4 \pi^{2}}\right)^{1 / 3} \simeq 1.7 \times 10^{8} \times\left(\frac{P_{\text {spin }}}{1 s}\right)^{2 / 3} \\
& \times\left(\frac{M}{1.4 \mathrm{M}_{\odot}}\right)^{1 / 3} \mathrm{~cm},
\end{aligned}
$$

where $P_{\text {spin }}$ is the stellar spin period of the neutron star. Again, since no pulsation has yet been detected from the neutron star in Sco X-1, its $P_{\text {spin }}$ is completely unknown. However, as it is a highly accreting LMXB system, its spin period is likely to be a few milliseconds, similar to that of observed recycled pulsars. Thus in our analysis, we consider the astrophysically plausible range of $f_{\text {spin }}\left(=1 / P_{\text {spin }}\right) \simeq$ $25-750 \mathrm{~Hz}$. Together with the assumption that $M \simeq$ 1.1-2.5 $\mathrm{M}_{\odot}$, the expected range of $r_{\mathrm{co}}$ corresponds to $r_{\mathrm{co}} \simeq 20-300 \mathrm{~km}$. Therefore, the very conservative astrophysical range of $\left(r_{m} / r_{\mathrm{co}}\right)$ for the neutron star in Sco X-1 lies between $\simeq 0.03-100$.

The amount of spin-wandering depends on the ratio of two characteristic length scales, $\left(r_{m} / r_{\mathrm{co}}\right)$, rather than each of them individually. Note that, Sco X-1 has been observed to be a Z-type LMXB, an actively accreting neutron star sources with mass accretion rate persistently close to Eddington luminosity ever since its detection. A large $\dot{M}$ will increase the neutron star's spin frequency and thus will decrease $r_{\mathrm{co}}$, resulting in increasing the ratio of $\left(r_{m} / r_{\mathrm{co}}\right)$. Therefore, large values of $\left(r_{m} / r_{\mathrm{co}}\right)$ are strongly favored for the neutron star in Sco X-1. However, persistent mass accretion is strongly disfavored for $\left(r_{m} / r_{\mathrm{co}}\right)>1[3,26]$, contrary to the observed persistent accretion rate in Sco X-1. It is also worth mentioning that, the spin-wandering effect is insignificant for small values of $\left(r_{m} / r_{\mathrm{co}}\right)$ while potentially important to candidate searches for large values. The most realistic astrophysical expected scenario for the neutron star in Sco X-1 corresponds to $\left(r_{m} / r_{\mathrm{co}}\right) \sim 1$. This case we refer to as the "realistic" scenario for our following study. In this paper, we also explore the very "pessimistic" scenario corresponding to $\left(r_{m} / r_{\mathrm{co}}\right) \sim 100$.

\section{Estimating moment of inertia $\left(I_{\text {spin }}\right)$ of the neutron star in Sco X-1}

The moment of inertia about the spin-axis $\left(I_{\text {spin }}\right)$ also plays an important role in our analysis, since it determines the angular acceleration due to the torque exerted on the neutron star. We considered a physically plausible range of $I_{\text {spin }}$ for a neutron star including rapid spin. We also note that $I_{\text {spin }}$ depends on the $\operatorname{mass}^{9}(M)$ and currently unknown

\footnotetext{
9In this paper, by "mass" we refer to "gravitational mass" for simplicity, unless otherwise specified.
}

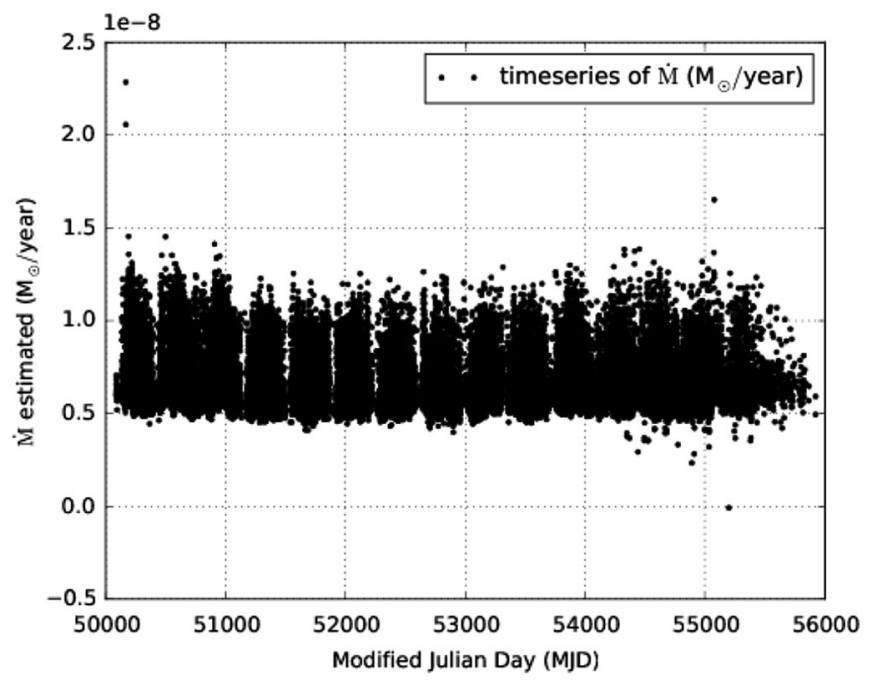

FIG. 3. The inferred time series of the mass accretion rate $\dot{M}(t)$ from Sco X-1 over approximately 15 years period in Modified Julian Day (MJD).

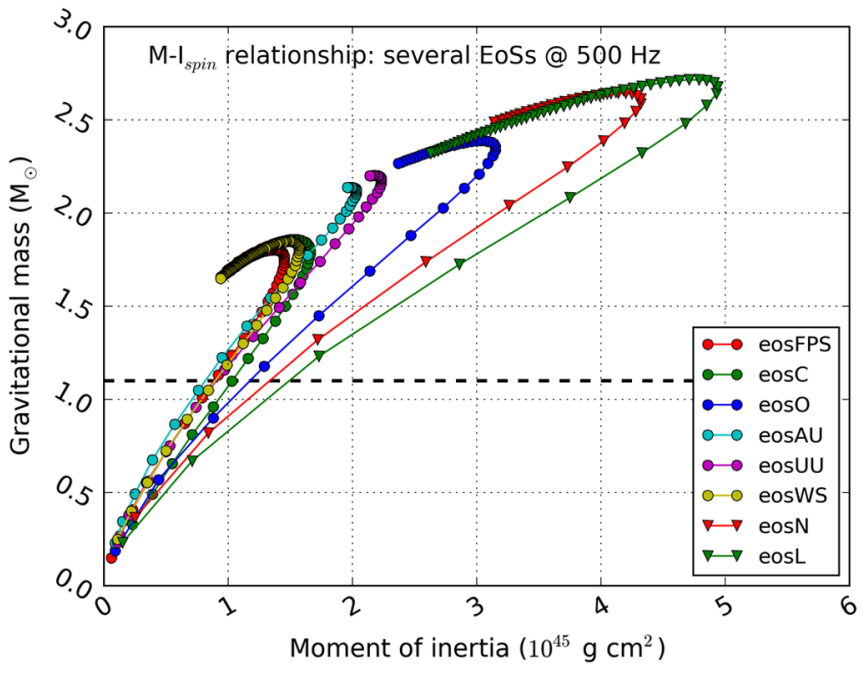

FIG. 4. The moment of inertia along the spin-axis $\left(I_{\text {spin }}\right)$ as a function of different gravitational masses of neutron star for a number of realistic candidate equations of state is shown. Note that, $I_{\text {spin }}$ of a neutron star depends not only on the nature of its equations of state, but also on its gravitational mass. The astrophysically plausible value of gravitational mass of a neutron star lies somewhere between the $1.1 \mathrm{M}_{\odot}$ (denoted with the horizontal black dashed-line) and the maximum gravitational mass allowed by the particular equation of state for stable configuration.

equation-of-state $(\mathrm{EoS})$ of neutron stars. Moreover, $I_{\text {spin }}$ can differ depending on whether the neutron star is rapidly spinning or in a static configuration.

For this purpose, we have numerically computed the moment of inertia along the spin-axis $\left(I_{\text {spin }}\right)$ in a fully general relativistic framework, following the procedure formulated in [27]. We have considered a number of 
realistic EoSs, from soft to stiff, for a neutron star over a range of stellar spin frequencies from non-spinning up to $750 \mathrm{~Hz}$.

In this paper, we consider a number of equations of state eosAU: WFF1 (denoted AV14 + UVII in Wiringa et al. [28]) matched to Negele-Vautherin; eosC: Bethe-Johnson model 1 (denoted EOS C in Arnett and Bowers [29]); eosFPS: by Lorenz, Ravenhall and Pethick [30]; eosUU: WFF2 (denoted UV14 + UVII in Wiringa et al. [28]) matched to Negele-Vautherin; eosWS: WFF3 (denoted UV14 + TNI in Wiringa et al. [28]) matched to eosFPS; eosO: by Bowers, Gleeson, and Pedigo (denoted EOS O in Arnett and Bowers [29]); eosN: Walecka-Serot (denoted EOS N in Arnett and Bowers [29]); eosL: by Pandharipande and Smith (mean field theory, denoted EOS L in Arnett and Bowers [29]). In Fig. 4, we show the mass vs moment of inertia curves for a number of equations of state for a stellar spin frequency of $500 \mathrm{~Hz}$. The physical range of $I_{\text {spin }}$ corresponds to $\sim 0.8-5.0 \times 10^{45} \mathrm{~g}-\mathrm{cm}^{2}$ for different values of mass, as well as different EoSs.

\section{NUMERICAL SIMULATION OF SPIN WANDERING}

The purpose of this analysis is to estimate the nature and degree of this spin-wandering behavior and to assess how continuous GW search pipelines are potentially affected by it. For this purpose, we need to simulate a number of spinwandering realizations spanning the entire range of the aforementioned astrophysically feasible parameter space. Moreover, since the spin-wandering behavior is stochastic in

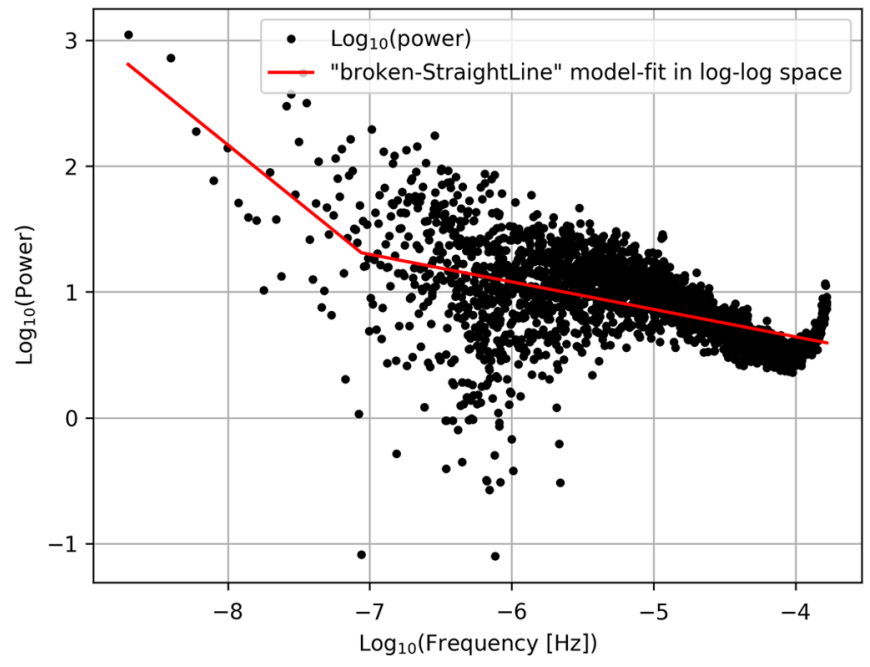

FIG. 5. The Lomb-Scargle periodogram computed from $R X T E / A S M$ lightcurve of Sco X-1 for $\sim 15$ year long observations. The periodogram was then fitted with a "broken straight line" model in the log-log space, representing a broken powerlaw model. The best fit model is shown with solid-red curve. We marginalize over the uncertainties in the estimated model parameters while performing the large-scale simulations.
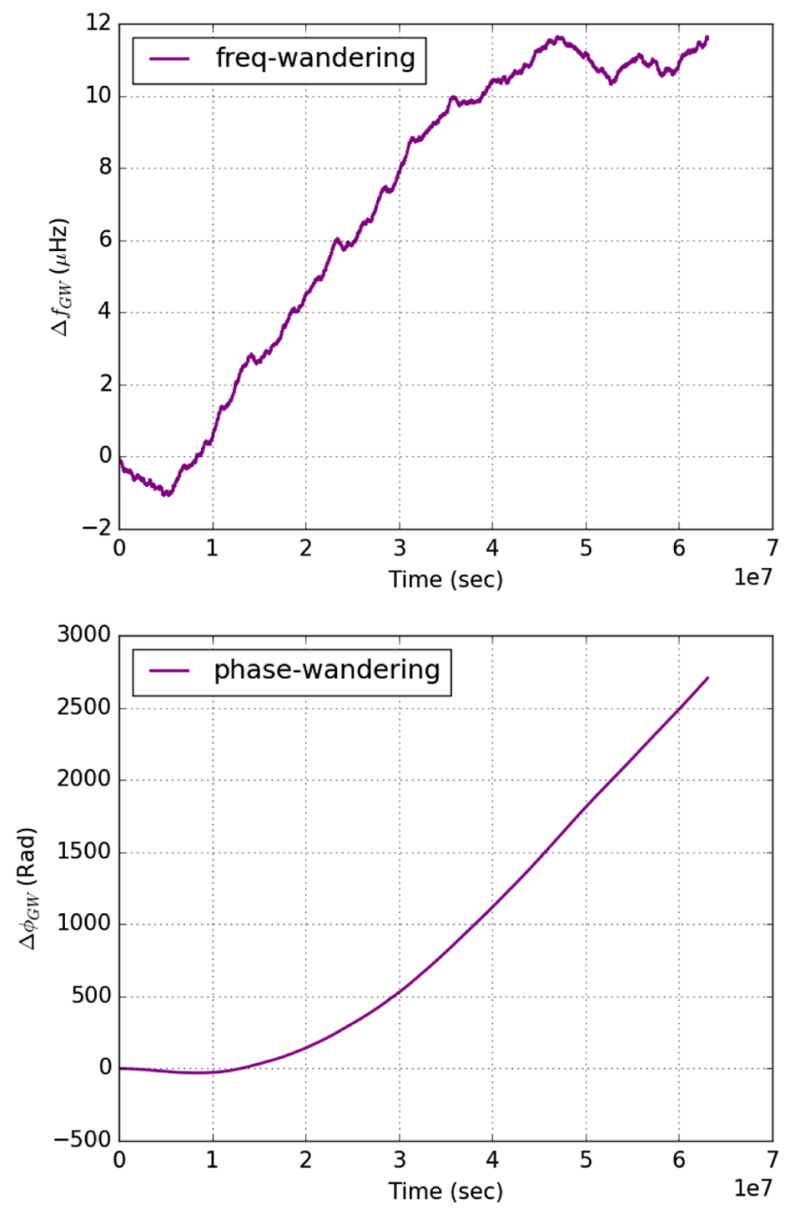

FIG. 6. The time evolution of a specific extreme realization of simulated spin-wandering time series for $f_{\text {spin }}=500 \mathrm{~Hz}, I_{\text {spin }}=$ $0.8 \times 10^{45} \mathrm{~g}-\mathrm{cm}^{2}$ and $r_{m} / r_{\text {co }}=100.0$. Top panel demonstrates the time series for instantaneous deviation of GW-frequency (i.e., $\left.2 \times f_{\text {spin }}\right)$. Bottom panel demonstrates the time series for cumulative deviation of GW-phase (i.e., $2 \times \phi_{\text {spin }}$ ).

nature, a statistical study over an ensemble of realizations is more desirable than any individual realization. We briefly describe the key steps of our simulations and analysis, below.

We computed the variability of the mass accretion rate in the frequency domain using the Lomb-Scargle periodogram, ${ }^{10}$ since the RXTE/ASM observations of Sco X-1 were extremely unevenly spaced in time. The computed Lomb-Scargle periodogram was then fitted with a brokenpower-law model as (see Fig. 5) as defined by,

$$
\begin{aligned}
P(f) & =A f^{-n_{1}} \quad \forall f \leq f_{b} \\
& =A f_{b}^{n_{2}-n_{1}} f^{-n_{2}} \quad \forall f>f_{b}
\end{aligned}
$$

where, $P(f)$ is the power at frequency $f, n_{1}$ is the powerlaw index at lower frequency $f \leq f_{b}, n_{2}$ is the powerlaw index at higher frequency $f>f_{b}$, and $f_{b}$ is the

\footnotetext{
${ }^{10}$ https://docs.scipy.org/doc/scipy-0.18.1/reference/generated/ scipy.signal.lombscargle.html.
} 

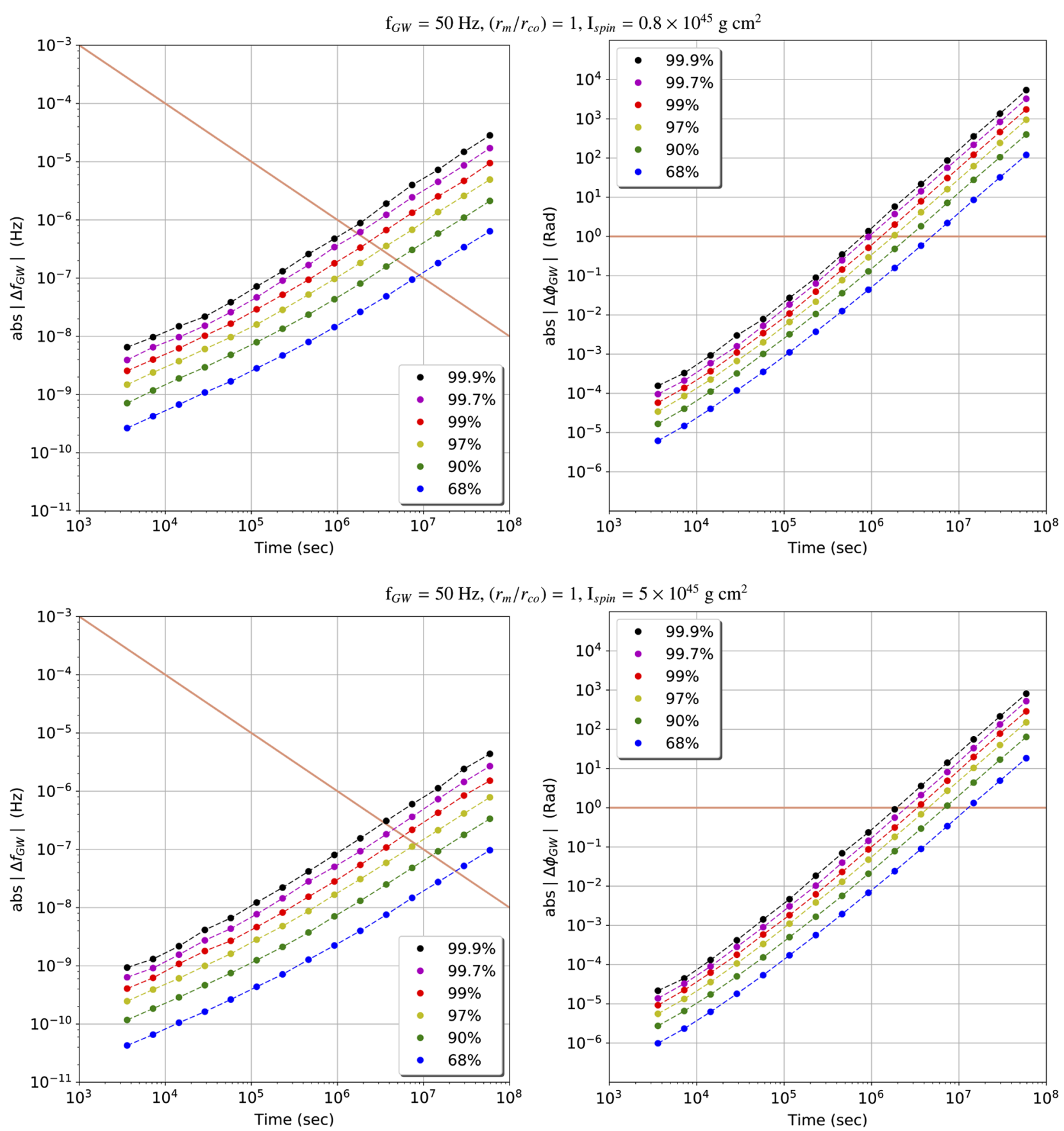

FIG. 7. This figure quantifies the statistical estimate of spin-wandering effect for the neutron star in Sco X-1. In each subplot different values of statistical confidence levels (see legends) are plotted that denote the cumulative probability of maximum frequency-wandering (left column) and phase-wandering (right column) effects. In this figure we take the unknown physical parameters of $f_{\text {spin }}=25 \mathrm{~Hz}$ and $r_{m} / r_{\mathrm{co}}=1$ (the realistic scenario); corresponding to 2,2-mode $\mathrm{GW}$-frequency $f_{\mathrm{GW}}=50 \mathrm{~Hz}$. The top row corresponds to the minimum theoretical value of moment of inertia, $I_{\text {spin }}=0.8 \times 10^{45} \mathrm{~g}-\mathrm{cm}^{2}$, while the bottom row corresponds to the maximum theoretical value of moment of inertia $I_{\text {spin }}=5 \times 10^{45} \mathrm{~g}-\mathrm{cm}^{2}$. For each case of a specific choices of $f_{\text {spin }},\left(r_{m} / r_{\text {co }}\right)$ and $I_{\text {spin }}$, we simulated 10,000 spinwandering realizations and estimated the amount of deviation in instantaneous $f_{\mathrm{GW}}$ and $\phi_{\mathrm{GW}}$ from their initial values at a number of statistical significance levels, $68 \%, 90 \%, 97 \%, 99 \%, 99.7 \%$ and $99.9 \%$ as a function of time. The absolute value of deviation in $f_{\mathrm{GW}}$ and $\phi_{\mathrm{GW}}$ for a given significance-level corresponds to the fraction of the spin-wandering realizations remaining within the respective range. Each of the orange straight lines for frequency-wandering cases (panels in the left column) denotes the frequency resolution as a function of time of integration of the signal. The orange straight-lines for phase-wandering cases (panels in the right column) denote phase mismatches of $1 \mathrm{rad}$ due to the spin-wandering effect. 

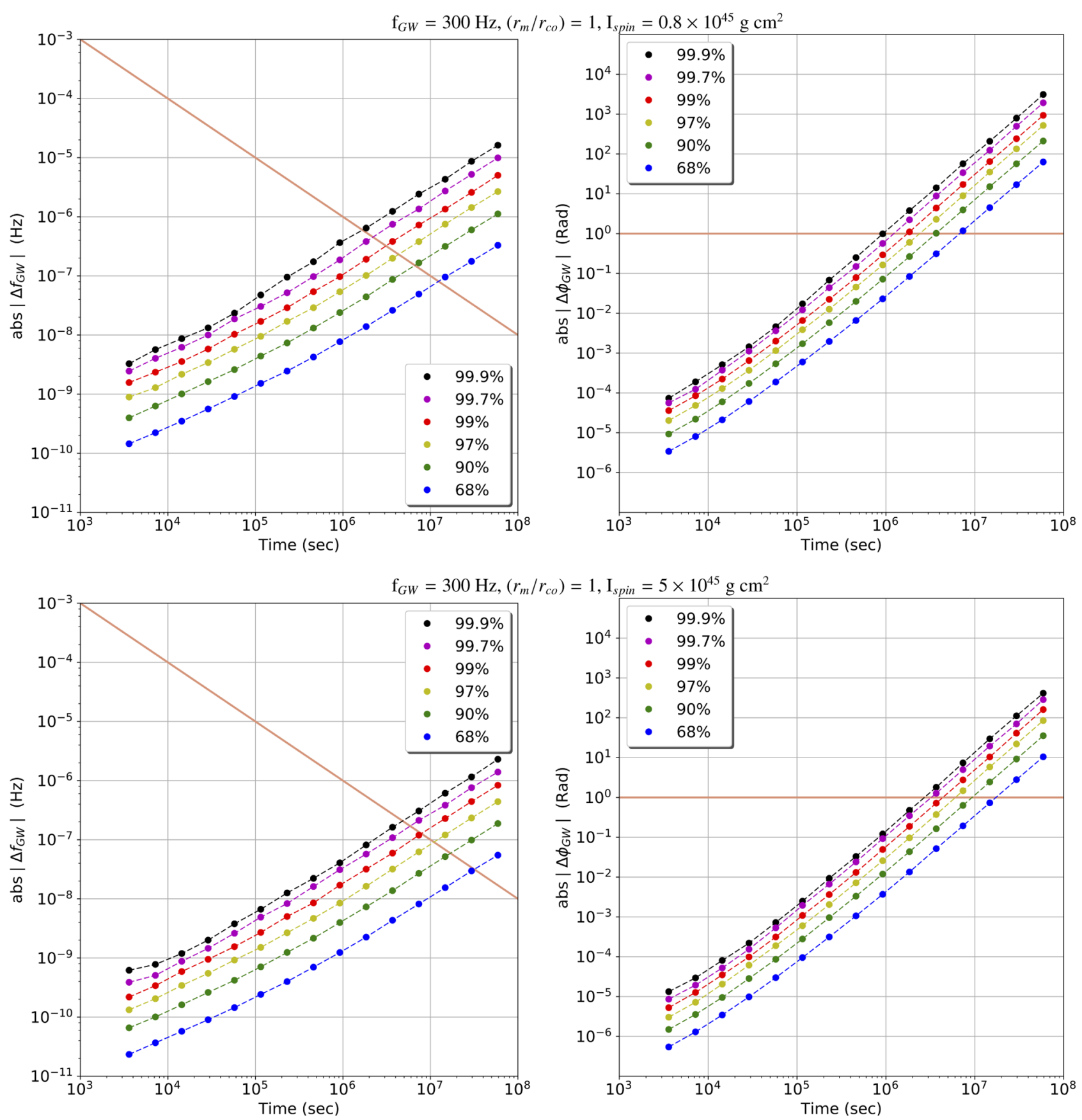

FIG. 8. This figure is similar to Fig. 7, but with different values of the unknown physical parameters. In this figure we take parameters of $f_{\text {spin }}=150 \mathrm{~Hz}$ and $r_{m} / r_{\mathrm{co}}=1$ (the realistic scenario); corresponding to 2,2-mode $\mathrm{GW}$-frequency $f_{\mathrm{GW}}=300 \mathrm{~Hz}$. The top row corresponds to the minimum theoretical value of moment of inertia, $I_{\text {spin }}=0.8 \times 10^{45} \mathrm{~g}-\mathrm{cm}^{2}$, while the bottom row corresponds to the maximum theoretical value of moment of inertia $I_{\text {spin }}=5 \times 10^{45} \mathrm{~g}-\mathrm{cm}^{2}$.

break frequency. The estimated parameter values (with their $1 \sigma$ error) of the broken-power-law model corresponds to $n_{1}=(0.91 \pm 0.12), n_{2}=(0.22 \pm 0.074), f_{b}=$ $10^{-7.06 \pm 0.10} \mathrm{~Hz}$ and $A=10^{-5.12 \pm 0.091}$ (count-rate) ${ }^{2} / \mathrm{Hz}$. It is worth mentioning that, there is an uprising trend in the estimated PSD at the very high-frequency end. We are not completely certain about its physical origin, and whether this is an observational/instrumental artifact. The time scale at which it tends to peak seems to be quite close to the $R X T E$ orbital period ( $\approx 90$ mins). However, this amount of power on the time scale of $\sim$ hour has a negligible contribution to the time-scale of $\sim$ day or longer, where the spin-wandering effect is most relevant to our analysis.

We have simulated a number of time series realizations of the RXTE/ASM photon count rate, applying an inverse Fourier transform of evenly-sampled frequency series data 

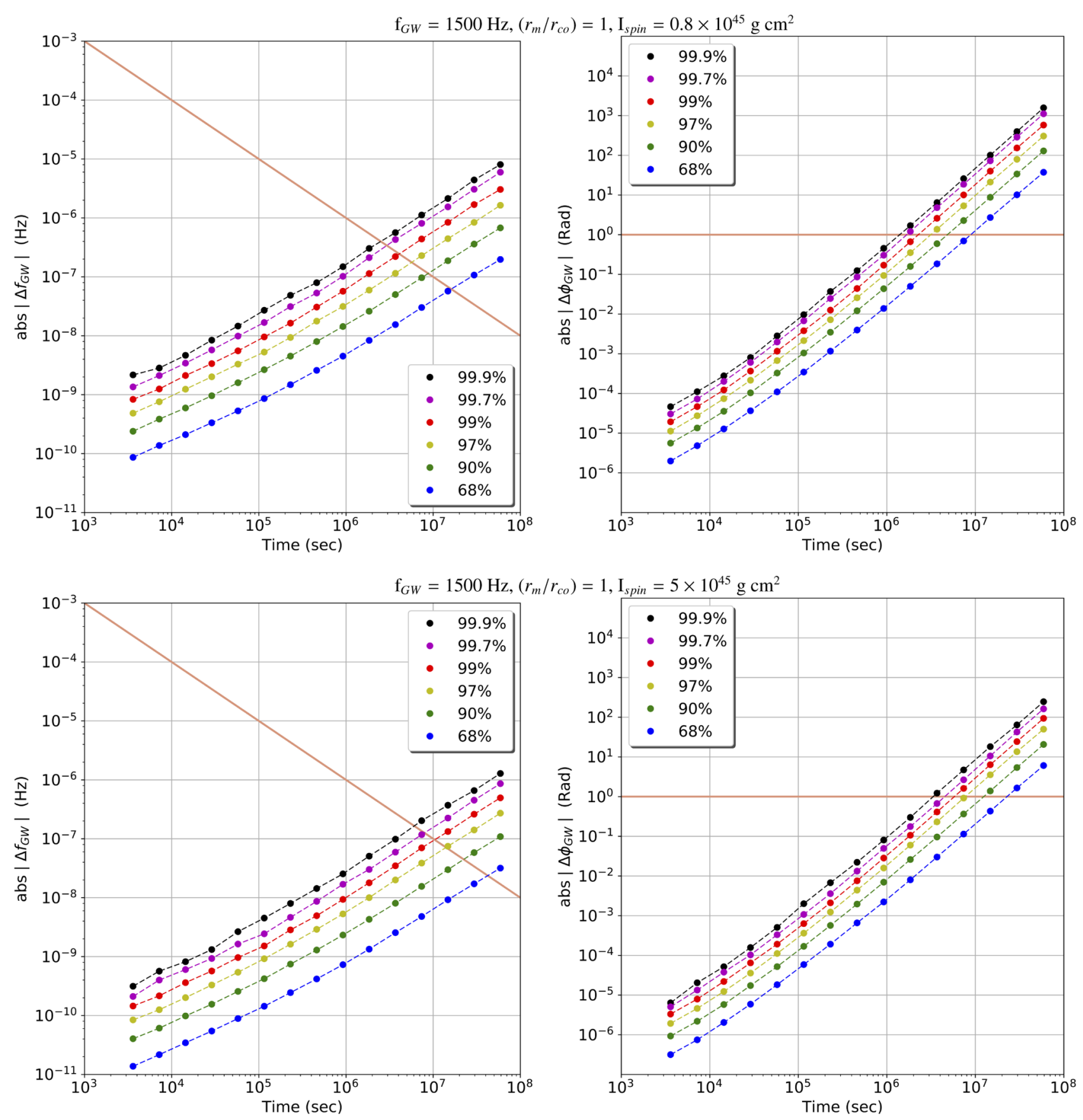

FIG. 9. This figure is similar to Fig. 7, but with different values of the unknown physical parameters. In this figure we take parameters of $f_{\text {spin }}=750 \mathrm{~Hz}$ and $r_{m} / r_{\mathrm{co}}=1$ (the realistic scenario); corresponding to 2,2-mode GW-frequency $f_{\mathrm{GW}}=1500 \mathrm{~Hz}$. The top row corresponds to the minimum theoretical value of moment of inertia, $I_{\text {spin }}=0.8 \times 10^{45} \mathrm{~g}-\mathrm{cm}^{2}$, while the bottom row corresponds to the maximum theoretical value of moment of inertia $I_{\text {spin }}=5 \times 10^{45} \mathrm{~g}-\mathrm{cm}^{2}$.

from this broken-power-law model in the frequency domain randomizing over the uncertainties in values of the estimated model parameters. For each time series generation we randomize the phase of each Fourier frequency to generate a statistically random time series obeying the same variability power density spectrum (PSD) as that estimated from x-ray data of RXTE observations. The corresponding $\dot{M}(t)$ time series is derived from the count rate time series following the calibration procedure discussed above. From each $\dot{M}(t)$ time series we compute the $\dot{f}(t)$ time series using Eq. (1). The $\dot{f}(t)$ time series is then integrated with respect to time to obtain the instantaneous frequency time series $f(t)$, and integrated once more to obtain the instantaneous phase time series $\phi(t)$. In this integration, we choose the first integration constant to be the equilibrium stellar spin frequency and the second 

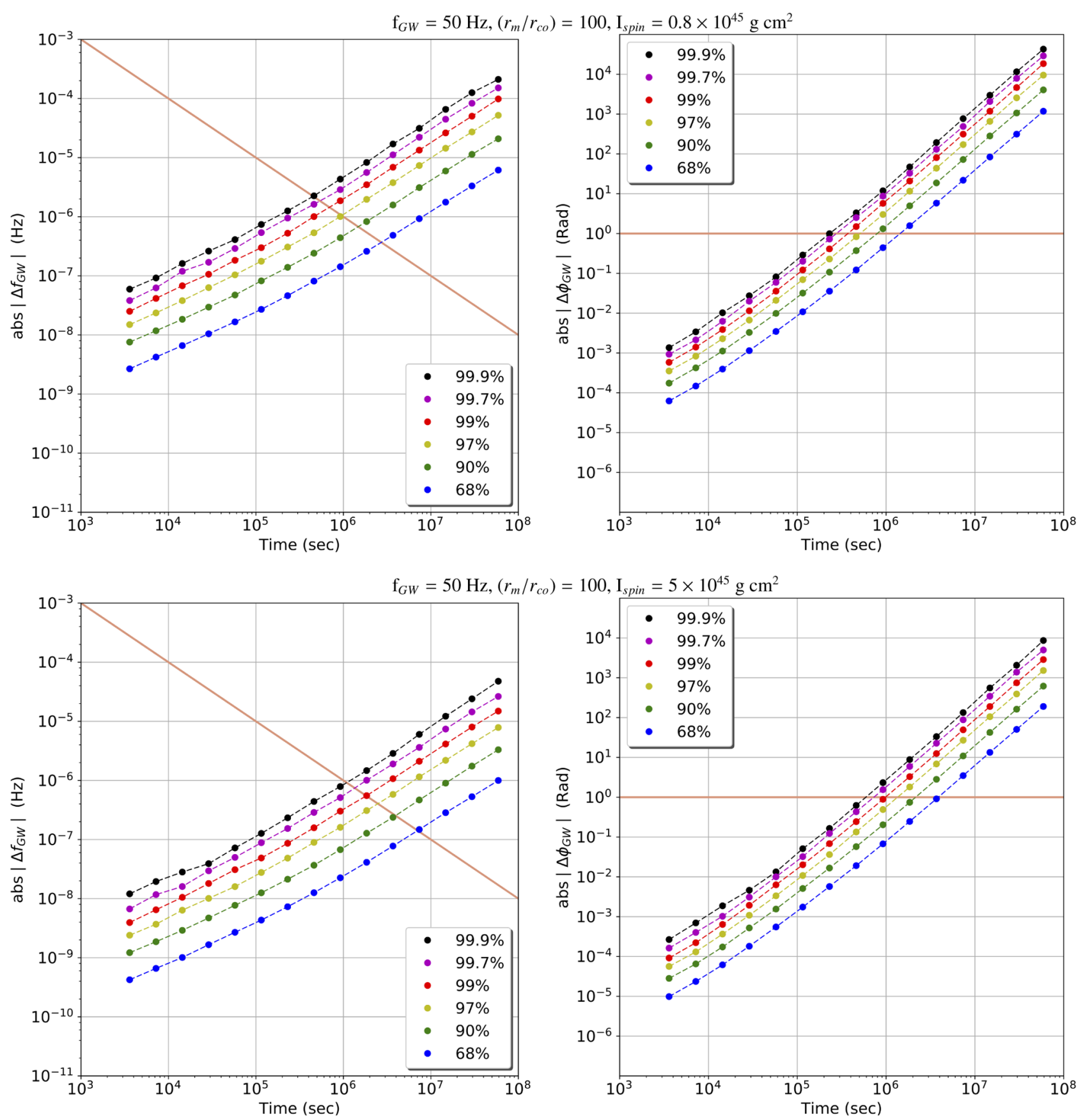

FIG. 10. This figure is similar to Fig. 7, but with different values of the unknown physical parameters. In this figure we take parameters of $f_{\mathrm{spin}}=25 \mathrm{~Hz}$ and $r_{m} / r_{\mathrm{co}}=100$ (the pessimistic scenario); corresponding to 2,2-mode GW-frequency $f_{\mathrm{GW}}=50 \mathrm{~Hz}$. The top row corresponds to the minimum theoretical value of moment of inertia, $I_{\text {spin }}=0.8 \times 10^{45} \mathrm{~g}-\mathrm{cm}^{2}$, while the bottom row corresponds to the maximum theoretical value of moment of inertia $I_{\text {spin }}=5 \times 10^{45} \mathrm{~g}-\mathrm{cm}^{2}$.

integration constant (reference phase) to be zero without any loss of generality.

One extreme example of an $f(t)$ time series (termed as "frequency wandering") and the corresponding $\phi(t)$ time series (termed as "phase wandering") are shown in Fig. 6. This particular example corresponds to one of the realizations from a large number of spin-wandering simulations. In this case, the unknown system parameters are taken to be $f_{\text {spin }}=500 \mathrm{~Hz}, I_{\text {spin }}=0.8 \times 10^{45} \mathrm{~g}-\mathrm{cm}^{2}$ and $r_{m} / r_{\mathrm{co}}=100.0$, corresponding to the above conservative scenario. From this example, it is clear that the phase can have an off-set of $\Delta \phi_{\mathrm{GW}} \approx 1$ radian in a time-scale of a single day in such an extreme case. We describe our extensive simulations of the spin-wandering effect over several astrophysically possible ranges of unknown parameters for the neutron star in Sco X-1. 

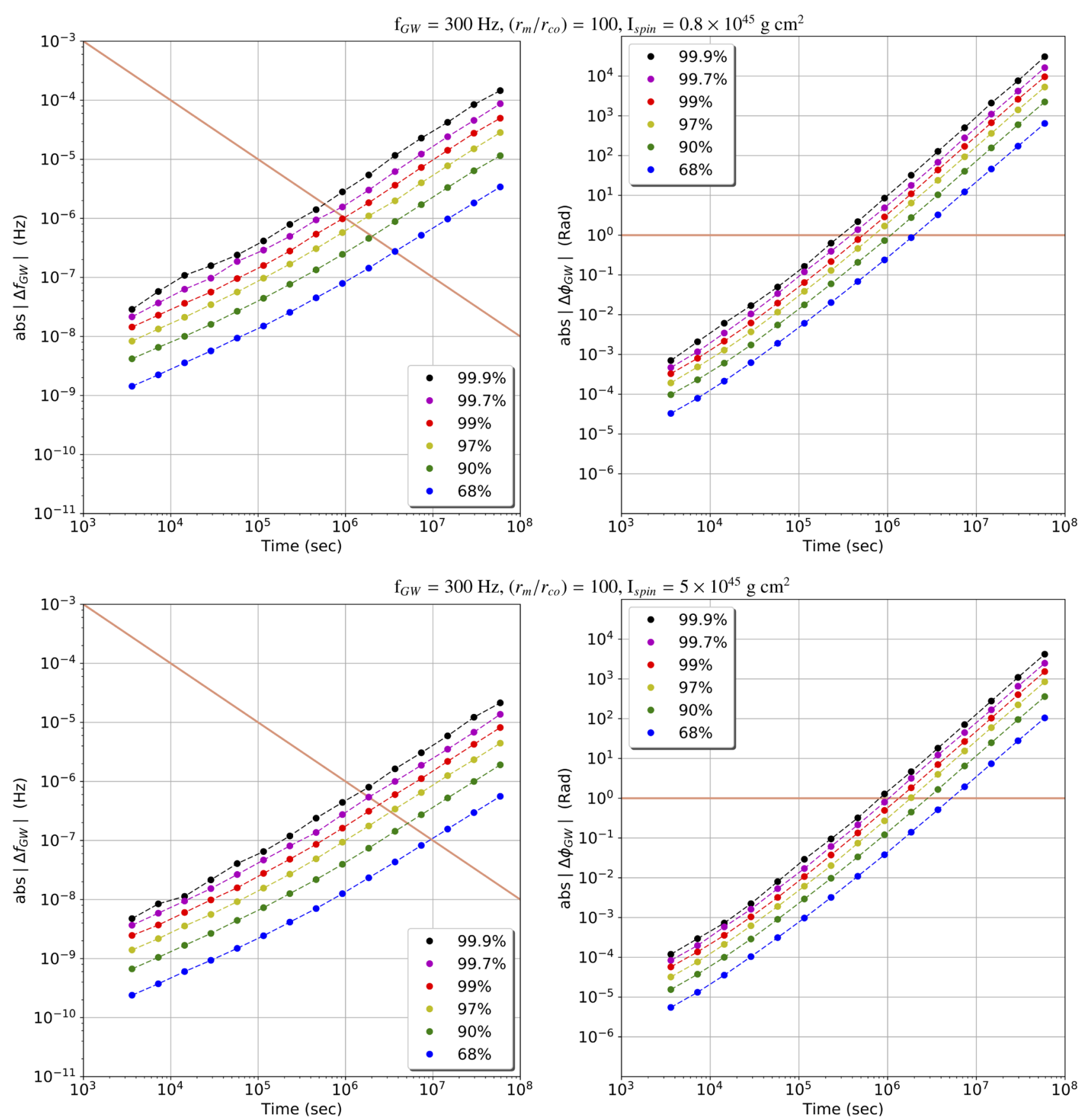

FIG. 11. This figure is similar to Fig. 7, but with different values of the unknown physical parameters. In this figure we take parameters of $f_{\text {spin }}=150 \mathrm{~Hz}$ and $r_{m} / r_{\mathrm{co}}=100$ (the pessimistic scenario); corresponding to 2,2-mode $\mathrm{GW}$-frequency $f_{\mathrm{GW}}=300 \mathrm{~Hz}$. The top row corresponds to the minimum theoretical value of moment of inertia, $I_{\text {spin }}=0.8 \times 10^{45} \mathrm{~g}-\mathrm{cm}^{2}$, while the bottom row corresponds to the maximum theoretical value of moment of inertia $I_{\text {spin }}=5 \times 10^{45}{\mathrm{~g}-\mathrm{cm}^{2}}^{2}$.

\section{A. Statistical behavior of spin wandering of the neutron star in Sco X-1}

Since accretion-induced spin wandering is stochastic, its effects are best studied statistically. Moreover, the values of several important quantities, such as spin frequency $\left(f_{\text {spin }}\right)$, the ratio of magnetic radius to corotation radius $\left(r_{m} / r_{\mathrm{co}}\right)$, and moment of inertia along the spin-axis $\left(I_{\text {spin }}\right)$, are unknown for Sco X-1 over broad ranges.
For this purpose, we have simulated several cases of spin-wandering effects, each case with a different set of astrophysical parameters, $f_{\text {spin }},\left(r_{m} / r_{\text {co }}\right)$ and $I_{\text {spin }}$. We have performed a large number of simulations for each set of assumed parameter values spanning their astrophysical expected range for the neutron star in Sco X-1. For each case of a specific choices of $f_{\text {spin }},\left(r_{m} / r_{\mathrm{co}}\right)$ and $I_{\text {spin }}$, we have simulated 10,000 spin-wandering realizations and 

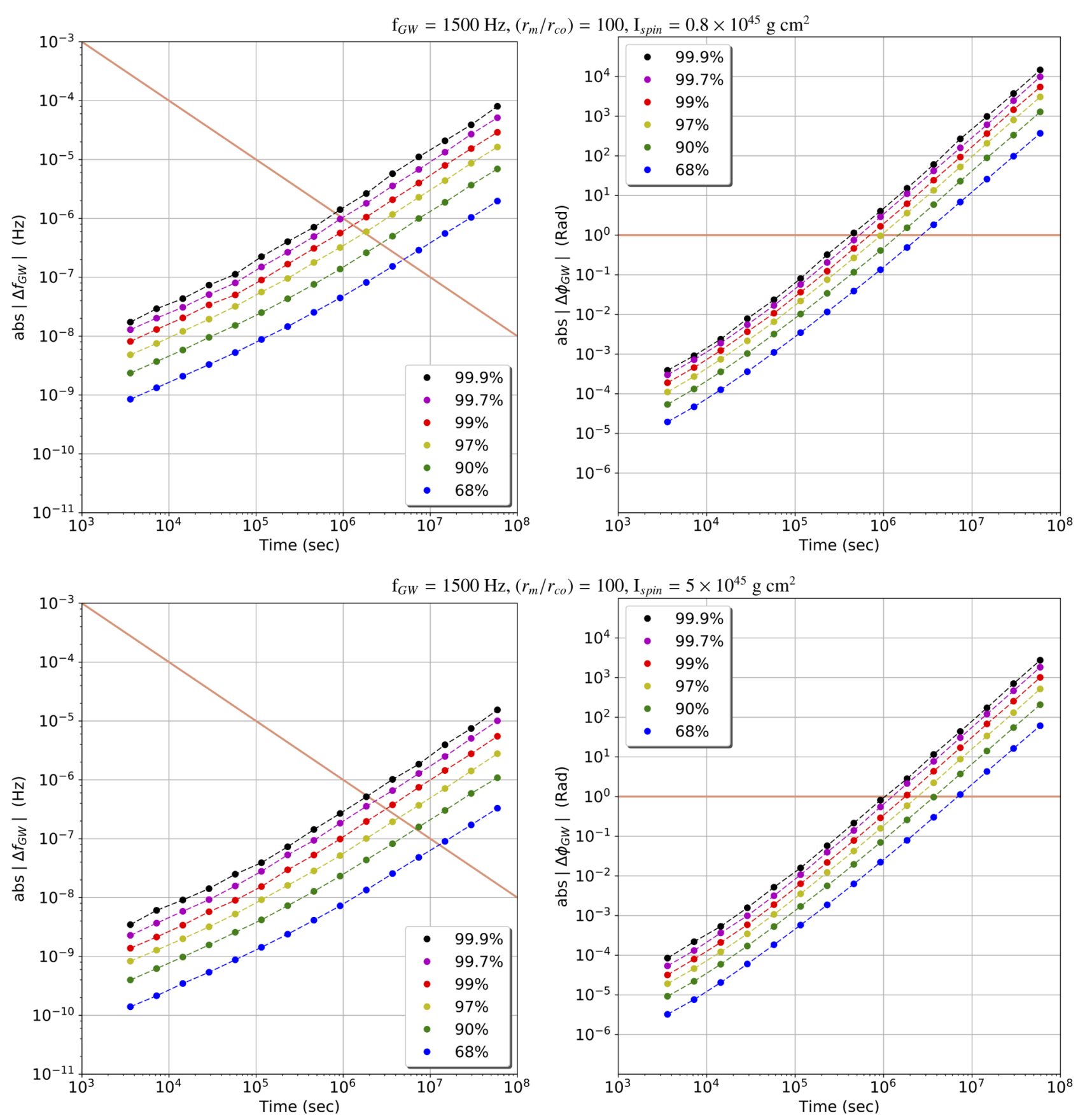

FIG. 12. This figure is similar to Fig. 7, but with different values of the unknown physical parameters. In this figure we take parameters of $f_{\text {spin }}=750 \mathrm{~Hz}$ and $r_{m} / r_{c o}=100$ (the pessimistic scenario); corresponding to 2,2-mode GW-frequency $f_{\mathrm{GW}}=1500 \mathrm{~Hz}$. The top row corresponds to the minimum theoretical value of moment of inertia, $I_{\text {spin }}=0.8 \times 10^{45} \mathrm{~g}-\mathrm{cm}^{2}$, while the bottom row corresponds to the maximum theoretical value of moment of inertia $I_{\text {spin }}=5 \times 10^{45} \mathrm{~g}-\mathrm{cm}^{2}$.

estimated the amount of deviation in instantaneous $f_{\mathrm{GW}}$ and $\phi_{\mathrm{GW}}$ from their initial values at a number of statistical significance levels, 68\%, 90\%, 97\%, 99\%, 99.7\%, and $99.9 \%$ as a function of time in the figures (Fig. 7 to Fig. 12). The absolute value of deviation in $f_{\mathrm{GW}}$ and $\phi_{\mathrm{GW}}$ for a given significance-level corresponds to the fraction of the spinwandering realizations remaining within the respective range.
We broadly classify all the simulations into two cases, the first one corresponding to $r_{m} / r_{\mathrm{co}}=1$, being the more realistic scenario. Next, we consider the extreme and pessimistic scenario corresponding to $r_{m} / r_{\mathrm{co}}=100$, leading to the maximum amount of spin-wandering effect for this source. We show the results of this large scale simulations in the Figs. 7, 8, 9 for the realistic scenario, and Figs. 10, 11, 12, for the pessimistic scenario. 
In these figures, we demonstrate both "frequency-wandering" and "phase-wandering" behavior quantitatively in statistical measure. We performed simulations for three values of continuous gravitational wave frequencies $\left(f_{\mathrm{GW}}\right)$, (i) at the low-frequency end $(50 \mathrm{~Hz})$, (ii) at a mid-frequency where the Advanced-LIGO and Advanced-Virgo noise sensitivity is good $(300 \mathrm{~Hz})$, and (iii) at the high-frequency end $(1500 \mathrm{~Hz})$, for each of the two scenarios. Moreover, for each of the aforementioned cases, we perform our simulations for the two extreme values of theoretically feasible moment of inertia along the spin-axis, $I_{\text {spin }}=0.8 \times$ $10^{45} \mathrm{~g}-\mathrm{cm}^{2}$ and $I_{\text {spin }}=5 \times 10^{45} \mathrm{~g}-\mathrm{cm}^{2}$, for the neutron star in Sco X-1.

\section{CONCLUSION AND DISCUSSION}

In this paper, we studied the spin-wandering effect of the neutron star in Sco X-1 using the x-ray observations (publicly available archival data from HEASARC facility of GSFC/NASA and MIT/XTE team) of the source with ASM and PCA instruments onboard the RXTE satellite. We developed and implemented the first methodology to infer the frequency wandering and phase wandering, collectively termed as "the spin-wandering effect," quantitatively with a range of astrophysically feasible parameter ranges. We have also studied the statistical behaviors of both frequency wandering and phase wandering.

The purpose of this study is not to perform a precise estimation of the spin-wandering effect nor to develop an improved theoretical model of accretion induced torque on the neutron star of an accreting LMXB system. Instead, we applied the existing standard physical model of accretioninduced torque by Bildsten et al. [4] on the accreting neutron star of Sco X-1 to infer the astrophysically expected range of the spin wandering.

We found the estimated effects of spin wandering can vary greatly, depending on the assumed astrophysical parameters of Sco X-1. In many cases, however, the degree of spin-wandering is significant and can plausibly degrade the sensitivity of some continuous gravitational wave search pipelines, particularly those that gain sensitivity from long coherence times and make rigid assumptions about the source frequency evolution.

In the plausible astrophysical scenario $r_{m} \sim r_{c o}$. In this case, the uncertainty in spin-wandering effect is dominated by $I_{\text {spin }}$ and stellar spin frequency of the neutron star in Sco $\mathrm{X}-1$. For the astrophysically extreme case of model parameters, the maximum amount of coherence time should be $\sim 2 \times 10^{6}$ seconds due to wandering frequency with a high degree of confidence level (99.9\%). However, the maximum coherence time should be limited to $\sim 8 \times 10^{5}$ seconds by the offset in phase due to spin-wandering effect (with $99.9 \%$ confidence level). For other possible values of $I_{\text {spin }}$ and $\mathrm{f}_{\text {spin }}$, the maximum duration of coherently integrable signal would be higher.

For the most pessimistic scenario, $\left(r_{m} / r_{c o}\right)$ could be as large as 100. In that case, the maximum amount of coherence time should be $\sim 2 \times 10^{5}$ seconds due to wandering frequency $(99.9 \%$ confidence level), whereas the maximum coherence time should be limited to $\sim 8 \times 10^{4}$ seconds by the offset in phase due to spin-wandering effect (99.9\% confidence level).

For any particular search pipeline, the maximum coherence time depends on the details of how sensitive it is to a frequency-offset and a phase-offset for a signal from the exact values of its search parameters. The performance of various pipelines will be performed in the upcoming mock data challenge for Sco X-1. As a consequence, it is important for GW search pipelines to evaluate their realistic sensitivity given the full range of astrophysically plausible spin-wandering effects presented in this paper.

The results of the first mock-data-challenge (MDC) specifically designed to test GW search pipelines in their ability to detect signals from Sco X-1 were published in [18]. This analysis did not explicitly include spin-wandering effects in the mock data analyzed by the challenge participants. We plan to use the spin-wandering simulation code developed for this paper in the generation of data for the second MDC. This will add an additional level of realism to the data and enable existing search pipelines to demonstrate their robustness against differing degrees of spin wandering.

Our analysis and modeling have some limitations, and a number of improvements could be made in the event of further relevant EM observations of Sco X-1. Here, we briefly mention a few key issues. First, we highlight our ignorance of the nominal spin frequency of Sco X-1. A future detection of coherent pulsations or burst oscillations would be immensely useful in narrowing down the uncertainty in several ways, including not only the approximate frequency band, but also in the estimation of the co-rotation radius $\left(r_{\mathrm{co}}\right)$, which affects the degree of spin wandering. There has been some recent activity, regarding deep searches of coherent pulsations in a number of accreting LMXBs (Messenger and Patruno [31], Patruno et al. [32]). Although it is difficult to predict the detection of pulsations from a source, it might be possible to detect a signal from Sco X-1 in the next few years, or possibly with the advent of next generation $\mathrm{x}$-ray satellites with much larger photon collecting areas.

Any determination of the magnetic field of the neutron star in Sco X-1 would also be useful for estimating the magnetic radius $\left(r_{m}\right)$ and thus allow us to better estimate the degree of expected spin wandering. Moreover, improved knowledge of the mass, and the correct EoS of the neutron star would also help improve the estimation of $I_{\text {spin }}$, which 
also affects the degree of spin wandering. Finally, note that we have ignored any possible "propeller effect" that may arise in disk-magnetospheric interaction in our analysis. In the propeller phase, accretion induced torque on the neutron star can significantly differ from standard accretion disk magnetospheric torque $[33,34]$ quantified by Eq. (1).

It is worth mentioning that if the neutron star in Sco X-1 is not in the torque-balance regime, that is, if the neutron star is either spinning-up or spinning-down steadily over an observation period, then a CW search pipeline will need to allow for a frequency derivative parameter. However, the very stochastic nature of frequency and phase evolution due to the spin-wandering affect the length of time over which we can coherently analyze the signal; that coherence time remains unchanged.

Similarly, in this paper, we assumed that the ellipticity of the neutron star in Sco X-1 does not change with time. If the ellipticity varied secularly, however, then the spin-down torque from gravitational radiation would vary, again potentially requiring an additional frequency derivative parameter.

In future, long duration $x$-ray observations, preferably at equally-sampled time intervals would be very useful. In our analysis, we used the publicly available RXTE/ASM data of Sco X-1 from the MIT/XTE team and the HEASARC facility of GSFC/NASA. The available observational data are extremely sparsely sampled, however. This prevented use of conventional Fourier transform methods to estimate the variability characteristics in the frequency domain. Instead, we used Lomb-Scargle periodograms which are subject to larger spectral leakage, leading to potential systematic bias in our analysis. Present and upcoming x-ray instruments, e.g., the Scanning Sky Monitor (SSM) onboard AstroSat, could be very useful, particularly the event mode data, containing information of individual photon arrival time, to mitigate this problem.

\section{ACKNOWLEDGMENTS}

This research has made use of data and software provided by the High Energy Astrophysics Science Archive Research Center (HEASARC), which is a service of the Astrophysics Science Division at NASA/GSFC and the High Energy Astrophysics Division of the Smithsonian Astrophysical Observatory. A. M. thanks Alan Levine, Tod Strohmayer and XTE team at MIT for sharing information on the RXTE/ASM data. The authors would also like to acknowledge valuable input from several members within LIGO Scientific Collaboration (LSC) and Virgo collaboration, and would particularly like to thank G. Ashton, G. Meadors, L. Sun, and J. T. Whelan for their comments on an early draft. A. M. is also thankful to R. Prix and H.-B. Eggenstein for very useful feedback to improve this paper. A. M. acknowledges support from the "SERB Start-Up Research for Young Scientists Scheme" project Grant No. SB/FTP/PS-067/2014, DST, India and from the Max Planck Partner Group at ICTS-TIFR. K. R. acknowledges support from the National Science Foundation (Grant No. PHY-1505932). The authors also gratefully acknowledge the Science and Technology Facilities Council of the United Kingdom. C. M. is supported by the Science and Technology Research Council (Grant No. ST/L000946/1). Most of the computational studies reported here were run on the ATLAS computing cluster at Max-Planck-Institut für Gravitationsphysik (Albert-Einstein-Institut), Hannover and computational cluster Mowgli and Alice at International Centre for Theoretical Sciences, TIFR, Bangalore. The authors gratefully acknowledge the support of the Max Planck Society and the State of Niedersachsen, Germany, for provision of computational resources. This paper has LIGO document number LIGO-P1700190.
[1] J. Papaloizou and J. E. Pringle, Mon. Not. R. Astron. Soc. 184, 501 (1978).

[2] R. V. Wagoner, Astrophys. J. 278, 345 (1984).

[3] L. Bildsten, Astrophys. J. Lett. 501, L89 (1998).

[4] L. Bildsten, D. Chakrabarty, J. Chiu, M. H. Finger, D. T. Koh, R. W. Nelson, T. A. Prince, B. C. Rubin, D. M. Scott, M. Stollberg, B. A. Vaughan, C. A. Wilson, and R. B. Wilson, Astrophys. J. Suppl. Ser. 113, 367 (1997).

[5] D. Chakrabarty, AIP Conf. Proc. 1068, 67 (2008).

[6] A. Patruno, B. Haskell, and N. Andersson, Astrophys. J. 850, 106 (2017).

[7] B. P. Abbott et al. (Virgo Collaboration and LIGO Scientific Collaboration), Phys. Rev. Lett. 116, 061102 (2016).
[8] B. P. Abbott et al. (Virgo Collaboration and LIGO Scientific Collaboration), Phys. Rev. Lett. 116, 241103 (2016).

[9] B. P. Abbott, R. Abbott, T. D. Abbott, M. R. Abernathy, F. Acernese, K. Ackley, C. Adams, T. Adams, P. Addesso, R. X. Adhikari et al., Phys. Rev. X 6, 041015 (2016).

[10] B. Abbott et al. (Virgo Collaboration), Phys. Rev. Lett. 118, 221101 (2017).

[11] B. P. Abbott, R. Abbott, T. D. Abbott, F. Acernese, K. Ackley, C. Adams, T. Adams, P. Addesso et al. (The LIGO Scientific Collaboration and the Virgo Collaboration), Phys. Rev. Lett. 119, 141101 (2017).

[12] B. P. Abbott, R. Abbott, T. D. Abbott, F. Acernese, K. Ackley, C. Adams, T. Adams, P. Addesso et al. (The LIGO 
Scientific Collaboration, and the Virgo Collaboration), Astrophys. J. 851, L35 (2017).

[13] B. P. Abbott, R. Abbott, T. D. Abbott, F. Acernese, K. Ackley, C. Adams, T. Adams, P. Addesso, R. X. Adhikari, V. B. Adya et al., Phys. Rev. Lett. 119, 161101 (2017).

[14] F. K. Lamb, in Proc. 23rd ESLAB Symp. Two Topics in X-Ray Astronomy, Volume 1: X Ray Binaries. Volume 2: $A G N$ and the X Ray Background, edited by J. Hunt and B. Battrick (Noordwijk, 1989).

[15] C. F. Bradshaw, E. B. Fomalont, and B. J. Geldzahler, Astrophys. J. Lett. 512, L121 (1999).

[16] P. Leaci and R. Prix, Phys. Rev. D 91, 102003 (2015).

[17] J. T. Whelan, S. Sundaresan, Y. Zhang, and P. Peiris, Phys. Rev. D 91, 102005 (2015).

[18] C. Messenger, H. J. Bulten, S. G. Crowder, V. Dergachev, D. K. Galloway, E. Goetz, R. J. G. Jonker, P. D. Lasky, G. D. Meadors, A. Melatos, S. Premachandra, K. Riles, L. Sammut, E. H. Thrane, J. T. Whelan, and Y. Zhang, Phys. Rev. D 92, 023006 (2015).

[19] J. Aasi, B. P. Abbott, R. Abbott, T. Abbott, M. R. Abernathy, F. Acernese, K. Ackley, C. Adams, T. Adams, P. Addesso et al., Phys. Rev. D 91, 062008 (2015).

[20] N. Andersson and K. D. Kokkotas, Int. J. Mod. Phys. D 10, 381 (2001).

[21] L. Lindblom, B. J. Owen, and G. Ushomirsky, Phys. Rev. D 62, 084030 (2000).
[22] W. H. G. Lewin, J. van Paradijs, and E. P. J. van den Heuvel, $X$-ray Binaries, edited by W. H. G. Lewin, J. van Paradijs, and E. P. J. van den Heuvel (Cambridge University Press, Cambridge, England, 1997).

[23] B. J. McNamara, J. Norwood, T. E. Harrison, J. Holtzman, R. Dukes, and T. Barker, Astrophys. J. 623, 1070 (2005).

[24] D. Mukherjee, P. Bult, M. van der Klis, and D. Bhattacharya, Mon. Not. R. Astron. Soc. 452, 3994 (2015).

[25] A. Papitto, A. D’Aì, T. Di Salvo, E. Egron, E. Bozzo, L. Burderi, R. Iaria, A. Riggio, and M. T. Menna, Mon. Not. R. Astron. Soc. 429, 3411 (2013).

[26] A. F. Illarionov and R. A. Sunyaev, Astron. Astrophys. 39, 185 (1975).

[27] G. B. Cook, S. L. Shapiro, and S. A. Teukolsky, Astrophys. J. 424, 823 (1994).

[28] R. B. Wiringa, V. Fiks, and A. Fabrocini, Phys. Rev. C 38, 1010 (1988).

[29] W. D. Arnett and R. L. Bowers, Astrophys. J. Suppl. Ser. 33, 415 (1977).

[30] C. P. Lorenz, D. G. Ravenhall, and C. J. Pethick, Phys. Rev. Lett. 70, 379 (1993).

[31] C. Messenger and A. Patruno, Astrophys. J. 806, 261 (2015).

[32] A. Patruno, K. Wette, and C. Messenger, arXiv:1709.10408.

[33] G. V. Ustyugova, A. V. Koldoba, M. M. Romanova, and R. V. E. Lovelace, Astrophys. J. 646, 304 (2006).

[34] M. M. Romanova, A. A. Blinova, G. V. Ustyugova, A. V. Koldoba, and R. V. E. Lovelace, arXiv:1704.08336. 